\title{
X-Ray and Neutron Tomography of Thermonuclear Plasmas
}

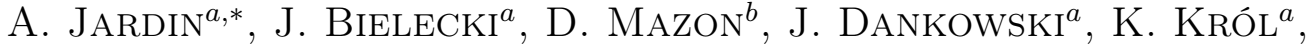

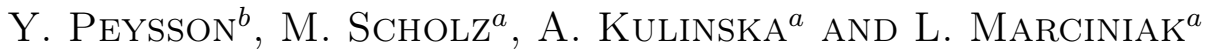 \\ ${ }^{a}$ Institute of Nuclear Physics, the Polish Academy of Sciences (IFJ PAN), \\ W.E. Radzikowskiego 152, PL-31342 Krakow, Poland \\ ${ }^{b}$ CEA, IRFM, F-13108 Saint Paul-lez-Durance, France
}

Doi: $10.12693 /$ APhysPolA.138.626

*e-mail: axel.jardin@ifj.edu.pl

\begin{abstract}
X-ray radiation and neutrons emitted from thermonuclear plasmas, like in tokamaks and plasma-focus devices, contain valuable information on the plasma dynamics and transport properties. Developing diagnostic tomography methods to retrieve the plasma emissivity from sparse measurement sets is thus an important issue. In this work, we report the development of a tomography method based on neural networks to achieve fast X-ray emissivity reconstructions in the prospect of real-time impurity control, with the first experimental test on a Tore Supra plasma subject to tungsten impurity injection. In parallel, preliminary investigations of neutron spectrum deconvolution on the PF-24 source from timeof-flight measurements using a Monte Carlo approach have been performed, including the assessment of robustness against experimental noise.
\end{abstract}

topics: X-ray, neutron, plasma tomography, tokamak

\section{Introduction}

$\mathrm{X}$-ray and neutron radiation emitted from thermonuclear fusion devices like tokamaks or plasmafocus (PF) devices can provide essential information on the plasma dynamics and properties, such as the electron/ion temperature, fusion reaction rate, impurity content or magnetohydrodynamic (MHD) activity [1]. The development of inversion methods in order to retrieve the plasma X-ray or neutron emissivity from sparse measurement sets is a key aspect to investigate. The forward problem can generally be expressed as

$$
f_{i}=\sum_{j} T_{i j} \varepsilon_{j}+\tilde{f}_{i},
$$

where $f_{i}$ denotes the $i$-th measurements, $\tilde{f}_{i}$ the associated experimental noise, $\varepsilon_{j}$ is the plasma emissivity in the $j$-th pixel and the transfer matrix elements $T_{i j}$ correspond to the response function of the detection system in the geometry of the considered tomographic problem. This expression indicates that a given inversion method can be generalized to different reconstruction problems, for instance spatial tomography or time-energy spectrum deconvolution. However, the inverse problem in (1) is mathematically ill-posed due to a sparse measurements set $\left\{f_{i}\right\}_{i=1 \ldots N}$ subject to systematic and statistical errors. Therefore, adapted reconstruction methods must be developed in order to select meaningful solutions, e.g. based on a maximum entropy algorithm [2], the Tikhonov regularization [3], a Monte Carlo approach [4] or machine learning [5].
The Institute for Magnetic Fusion Research (IRFM) and the Institute of Nuclear Physics of the Polish Academy of Sciences (IFJ PAN) conduct combined activities in this field under the French-Polish bilateral programme POLONIUM. The general goal of the collaboration is the development of methods of thermonuclear plasma diagnostic applied to current drive phenomena, MHD instabilities and impurity transport.

The goal of this contribution is to present recent results of these ongoing studies. In the second section of the article, special focus is put on a tomography method based on neural networks [6] that has been developed to achieve fast X-ray reconstructions with real-time impurity control capabilities [7] and that is tested here on experimental data from a Tore Supra (TS) plasma subject to a tungsten impurity injection. In the third section, preliminary investigations of neutron spectrum deconvolution on the PF-24 from time-of-flight (ToF) synthetic measurements using a Monte Carlo (MC) approach are reported. This study is a step towards experimental sessions being prepared in order to assess the correlation between the pinch disruption, X-ray emission and emitted neutron spectrum during PF24 plasma discharges. Finally, conclusions and perspectives of this work are drawn in the last section.

\section{Tokamak X-ray tomography}

A tokamak plasma tomography method based on neural networks has recently been developed to achieve fast soft X-ray (SXR) reconstructions with 


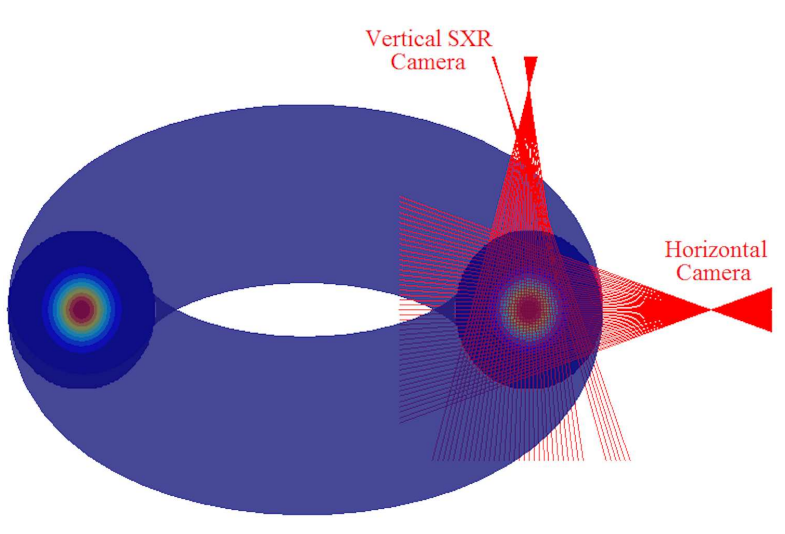

Fig. 1. Layout of the soft X-ray detection system of the Tore Supra tokamak.

real-time capabilities. Preliminary studies were performed using artificial data to assess the reconstruction capabilities [6]. Here, the neural network approach is validated against significant perturbative noise $-5 \%$ with a Gaussian probability distribution - in measurements and applied on TS experimental SXR signals collected during a tungsten (W) impurity injection by a laser blow-off system. The SXR detection system of TS, depicted in Fig. 1, is equipped with two fan-beam cameras composed of a set of silicon diodes measuring the line-integrated profiles of the plasma SXR emission in the range of $3-25 \mathrm{keV}$.

The neural network considered here is composed of two hidden fully-connected sigmoid neuron layers of size $30 \times 30$ with an input layer of 69 neurons, which is equal to the number of SXR channels used for the reconstruction, and an output layer to produce tomograms of size $50 \times 50$ pixels. The network parameters - weights and biases of each artificial neuron - are trained using a large database of artificial emissivity patterns and their associated synthetic measurements in order to minimize the global training loss that assesses the neural network performances [6]. The training loss is defined by the cost function (mean square error):

$$
C(\boldsymbol{w}, b)=\frac{1}{2 n} \sum_{\boldsymbol{x}}[y(\boldsymbol{x})-a(\boldsymbol{x}, \boldsymbol{w}, b)]^{2},
$$

where $(\boldsymbol{w}, b)$ are the weights and biases of the sigmoid neurons of the network, $(\boldsymbol{x}, y(\boldsymbol{x}))$ denotes a training sample - tomogram and associated measurements - and $a(\boldsymbol{x}, \boldsymbol{w}, b)$ is the output of the network. The training process is depicted in Fig. 2, where $90 \%$ of the data were used for training and $10 \%$ were kept outside of the training set for validation purposes once the neural network training is completed.

The reconstruction capabilities of the neural network are tested with artificial emissivity patterns taken from the validation set with noisy measurements as presented in Fig. 3. Then, as the first experimental test, the neural network is applied

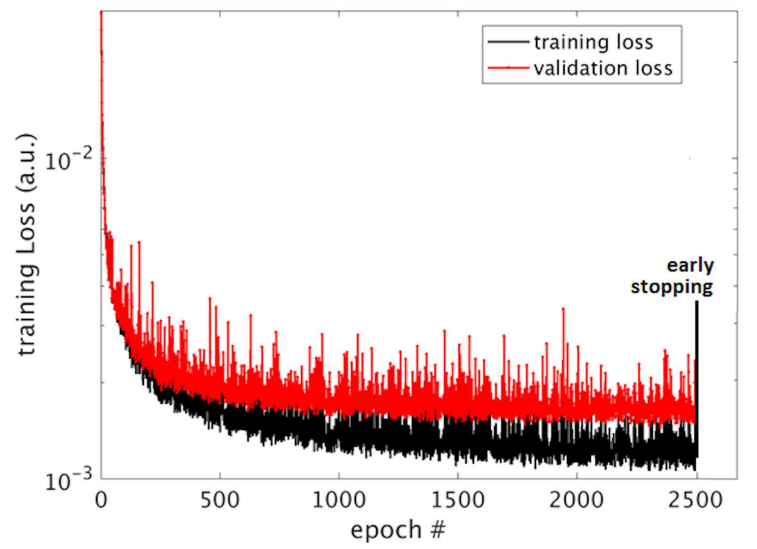

Fig. 2. Evolution of the training loss value of the neural network over the training process.

to the plasma discharge TS\#46564 at the time $t=18.2 \mathrm{~s}$, i.e., during the $\mathrm{W}$ impurity injection. A background subtraction technique during the injection time allows to extract the $\mathrm{W}$ contribution to the SXR signal. The tomograms in Fig. 4 are found to be consistent with former tomographic inversions performed using the traditional Tikhonov regularization method [3]. In particular, the in-out SXR asymmetry observed during the $\mathrm{W}$ injection is retrieved. However, the neural network is able to perform the inversions in a typical time $<0.1 \mathrm{~ms}$, i.e., more than two orders of magnitude faster and compatible with real-time control. This encouraging result should nevertheless be extended to other discharges to fully conclude about the extrapolability of the technique.

\section{Plasma-focus - Monte Carlo approach}

\subsection{Method}

In PF systems operated with deuterium, the dominant fusion reaction is

$$
{ }_{1}^{2} \mathrm{D}+{ }_{1}^{2} \mathrm{D} \rightarrow{ }_{2}^{3} \mathrm{He}+{ }_{0}^{1} \mathrm{n},
$$

releasing the $\mathrm{He}$ atom of the energy $\sim 0.82 \mathrm{MeV}$ and neutrons with the kinetic energy of $2.45 \mathrm{MeV}$ which can be detected by properly designed neutron diagnostics [8]. For such typical kinetic energies, the neutron velocity is of the order of $v \approx 2 \times 10^{7} \mathrm{~m} / \mathrm{s}$. Therefore, $v / c \approx 0.1$ at the frontier between classical and relativistic limits, where $c$ is the speed of light in vacuum. Hence, the goal is to unfold the neutron time-energy emitted spectrum using ToF measurements from a set of several detectors placed at different distances from the neutron source [9-11]. Indeed, the emitted timeenergy neutron spectrum can vary and provide valuable information on the plasma pinch dynamics, e.g. the pinch compression phase, acceleration mechanisms, thermal spectrum broadening or neutron scattering. The geometry of this problem is depicted in Fig. 5. 
(a)
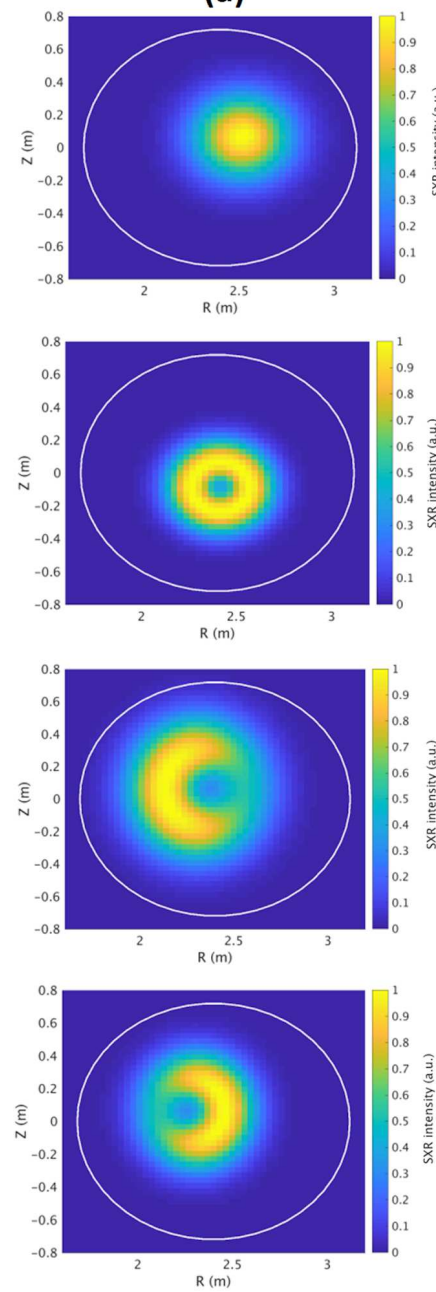

(b)
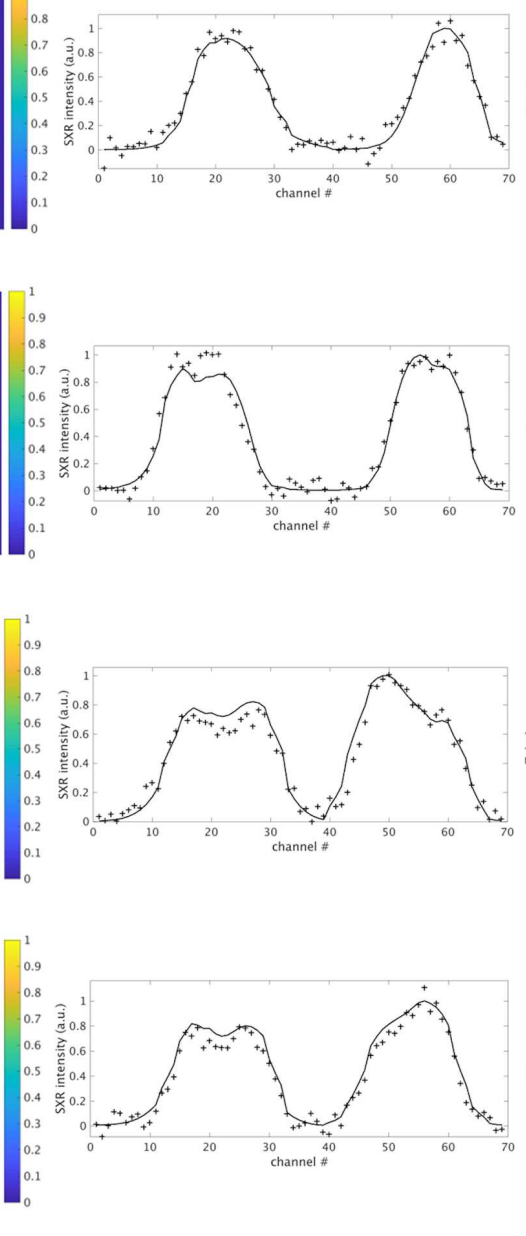

(c)
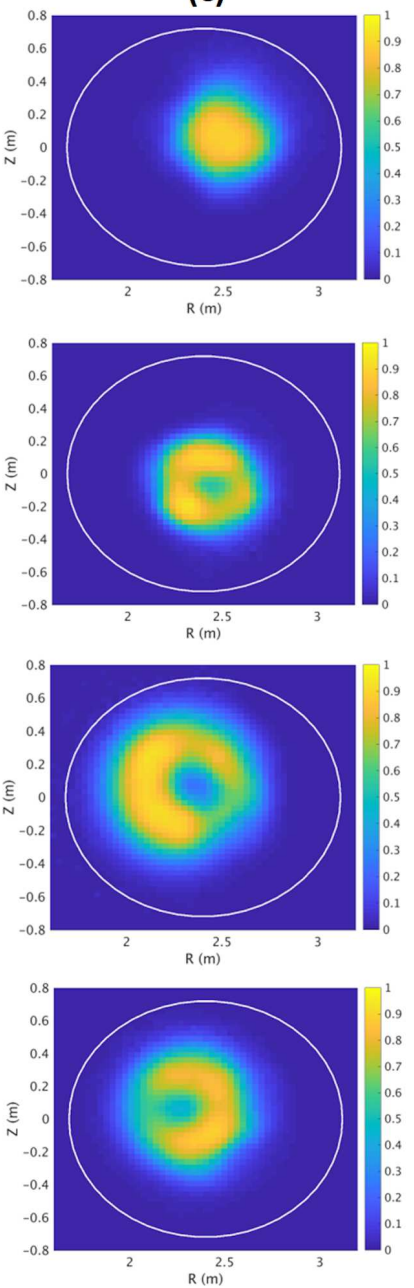

Fig. 3. (a) Artificial 2D emissivity phantoms. (b) Associated noisy line-integrated measurements (black crosses) and their retrofits (black line). (c) Tomograms reconstructed by the neural network.

(a)

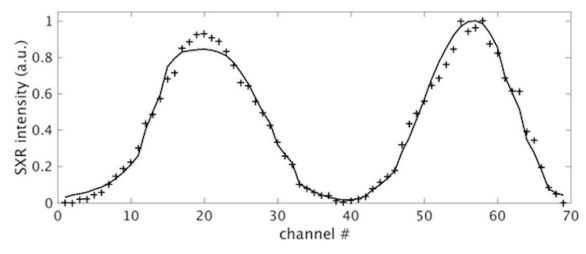

(b)

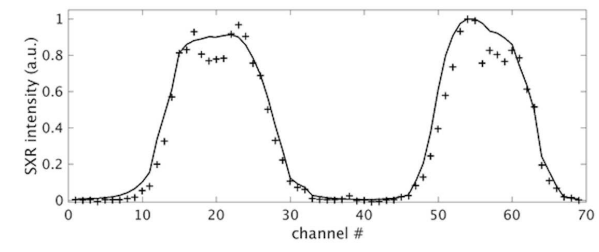

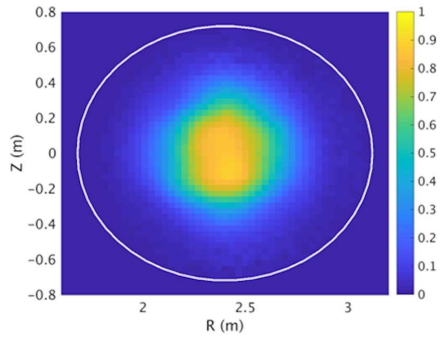

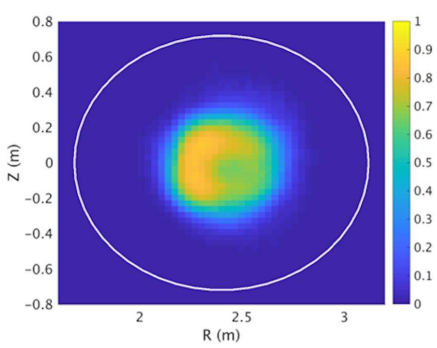

Fig. 4. Left: SXR line-integrated measurements (black crosses) and their retrofits (black line). Right: associated $2 \mathrm{D}$ reconstructed emissivity for TS\#46564 (a) just before the $\mathrm{W}$ injection at $t=18.2 \mathrm{~s}$, (b) just after the $\mathrm{W}$ injection with background subtraction. The emissivity fields and measurements are normalized between 0 and 1 to be used as inputs and outputs of the neural network. 


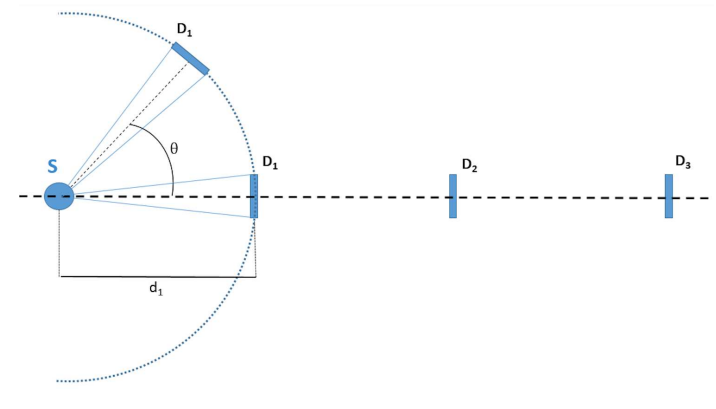

Fig. 5. Scheme of a set of ToF detectors $D_{j}$ positioned at different distances $d_{j}$ from the neutron source $S$ (plasma-focus, $\mathrm{PF}$ ), where $\theta$ is the detector angle with respect to the $\mathrm{PF}$ axis.

The neutron kinetic energy is expressed as $E=m_{0} c^{2}(\gamma-1)$, where $m_{0}$ is the neutron mass at rest and $\gamma=1 / \sqrt{1-v^{2} / c^{2}}$ is the Lorentz factor. Hence, the neutron velocity is

$$
v(E)=c \sqrt{1-\frac{1}{\left(1+\frac{E}{m_{0} c^{2}}\right)^{2}}} .
$$

A synthetic diagnostic can be created assuming that the signal $S_{d, \theta}(t)$, measured by a ToF detector placed at the distance $d$ and angle $\theta$ with respect to the PF-24 source, can be approximated by

$$
\begin{aligned}
& S_{d, \theta}(t)=\frac{C A_{D}}{4 \pi d^{2}} \int_{0}^{\infty} P(E) \\
& \quad \times f(t-d / v(E), E, \theta) \mathrm{d} E,
\end{aligned}
$$

where $C$ denotes the calibration coefficient specific to the detection system, $A_{D}$ - the effective collection area of the detector, $P(E)$ - the detection probability of a neutron of energy $E$ and $f(t, E, \theta)$ - the neutron spectrum emitted from the source (plasma pinch). In this preliminary work, we study the reconstruction capabilities of a set of ToF detectors without taking into account the spectral response of the detector. Therefore, hereafter $P(E)=1$ and $C=1$ will be assumed with the detector signal in arbitrary units. The energy resolution of the ToF detection system is given by

$$
\frac{\Delta E}{E}=\frac{2 \Delta t}{\tau},
$$

where $\Delta t$ is the neutron emission duration and $\tau=d / v$ is the neutrons ToF. In the specific case of the PF-24 device, the maximum source-detector distance is $d=10 \mathrm{~m}$, implying $\tau \approx 500 \mathrm{~ns}$ while the typical neutron emission duration $\Delta t \approx 50 \mathrm{~ns}$. Therefore, an energy resolution of $\Delta E / E \approx 20 \%$ can be expected.

\subsection{Neutron spectrum deconvolution}

The method developed and used for the deconvolution of the neutron spectrum relies on the Monte Carlo (MC) algorithm and is presented in Fig. 6. It is composed of the following steps:

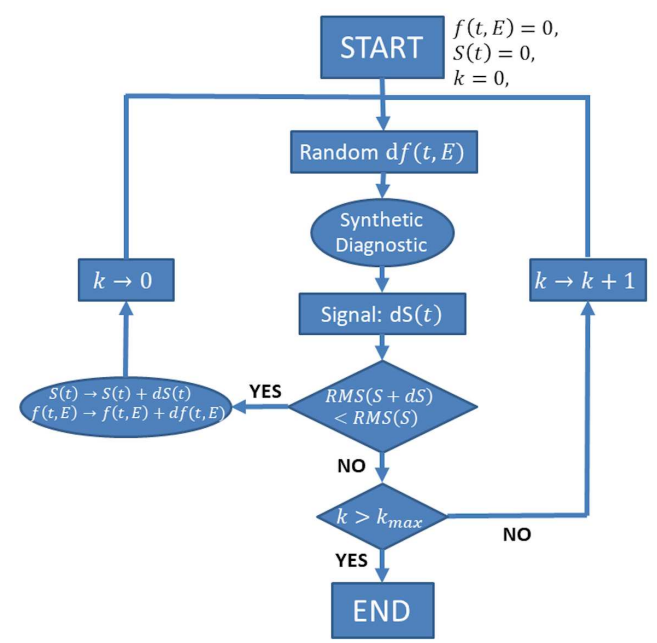

Fig. 6. Diagram of the Monte Carlo procedure for neutron spectrum deconvolution.

1. In the initial state, the reconstructed spectrum $f(t, E)$ and the associated ToF signal $S(t)=\left\{S_{j}(t)\right\}_{j=1 \ldots N}$ of the $N$ detectors are set to zero. The iteration index $k$ is set to zero.

2. A small element of neutron spectrum d $f(t, E)$ is randomly selected and the associated ToF signal increment $\mathrm{d} S(t)$ is computed.

3 . If the root mean square (RMS) error between the original signal $S_{0}(t)$ and the new reconstructed signal $S(t)+\mathrm{d} S(t)$ decreases, as defined in (7), the small increments $\mathrm{d} f(t, E)$ and $\mathrm{d} S(t)$ are added to the reconstructed neutron spectrum and ToF signal, respectively, and the variable $k$ is reset to zero. Otherwise, the variable $k$ is incremented, $k \rightarrow k+1$ :

$$
\operatorname{RMS}(S)=\sqrt{\sum_{j} \int\left(S_{j}(t)-S_{0, j}(t)\right)^{2} \mathrm{~d} t} .
$$

4. The two steps (1) and (2) are repeated iteratively until $k>k_{\max }$, for which the reconstruction process stops. The $k_{\max }$ value should be high enough, typically $k_{\max }>10^{4}$ in this work, to ensure the convergence of the algorithm.

An additional refinement is added to the deconvolution method. First, the Monte Carlo process is applied once assuming a uniform probability over the whole reconstruction range for the selection of $\mathrm{d} f(t, E)$, as described above. Then, the Monte Carlo algorithm is repeated a few times by replacing the uniform probability map with the reconstructed spectrum obtained at the last step, after convolution with a Gaussian function in order to smooth residual artefacts. This prior probability distribution allows preconditioning of the problem and notably increases the quality of the reconstruction. 
3.3. Dirac-like spectrum deconvolution in the one-detector case

Considering only one detector at a distance $d=2$, $d=5$ or $d=10 \mathrm{~m}$ along the axis of the PF device (for $\theta=0$ ), it is first assumed that the neutron emitted spectrum is a small rectangular function in time and energy and that it can be approximated by the Dirac function

$$
f\left(t, E_{\mathrm{kin}}, 0\right)=N \delta\left(t-t_{0}\right) \delta\left(E-E_{0}\right)
$$

where $N$ is the normalization factor to recover the total neutron rate. In this case, the predicted synthetic signal measured by the ToF detector will be

$$
\begin{gathered}
S(t, d)=\frac{N A_{D}}{4 \pi d^{2}} \int_{0}^{+\infty} \delta\left(E-E_{0}\right) \\
\times \delta\left(t-t_{0}-\frac{d}{v(E)}\right) \mathrm{d} E .
\end{gathered}
$$

Therefore

$$
S(t, d)=\frac{N A_{D}}{4 \pi d^{2}} \delta\left(t-\frac{d}{v\left(E_{0}\right)}-t_{0}\right)
$$

which corresponds to the Dirac function in the detector time trace, at the time $t=d / v\left(E_{0}\right)+t_{0}$. The detector time traces resulting from (5) for a small rectangular emitted spectrum are displayed in Fig. 7 for the three considered distances. The observed signal spreading is due to the small energy extension of the emitted spectrum, see Fig. 8a.

In the opposite way, still considering one detector only, the Dirac function in the detector time trace at any time $\tau$ is potentially associated with all tuples $(t, E)$ of the neutron spectrum satisfying the condition $\tau=d / v(E)+t$ or $v(E)=d /(\tau-t)$. Using (4), it corresponds to the curve

$$
E(t)=\frac{m_{0} c^{2}}{\sqrt{1-\frac{d^{2}}{c^{2}(\tau-t)^{2}}}}-m_{0} c^{2} .
$$

The preliminary test of deconvolution of a small rectangular spectrum in the one-detector case is presented in Fig. 8, together with the Dirac limit case (red line) obtained in (11). It is shown that one detector is not sufficient to reconstruct the neutron spectrum. However, the simultaneous use of several

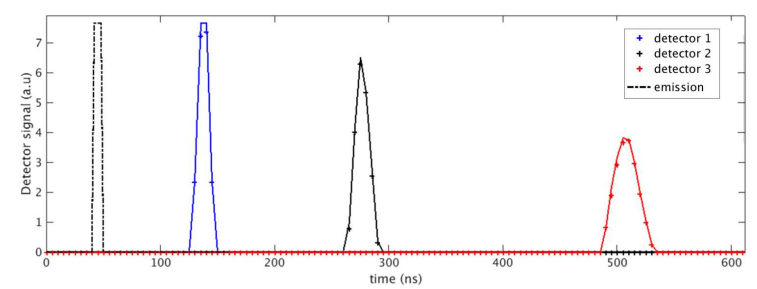

Fig. 7. ToF synthetic signals from the Dirac-like neutron source (in black dashed line) at distances of $2 \mathrm{~m}$ (in blue), $5 \mathrm{~m}$ (in black) and $10 \mathrm{~m}$ (in red) from PF-24.
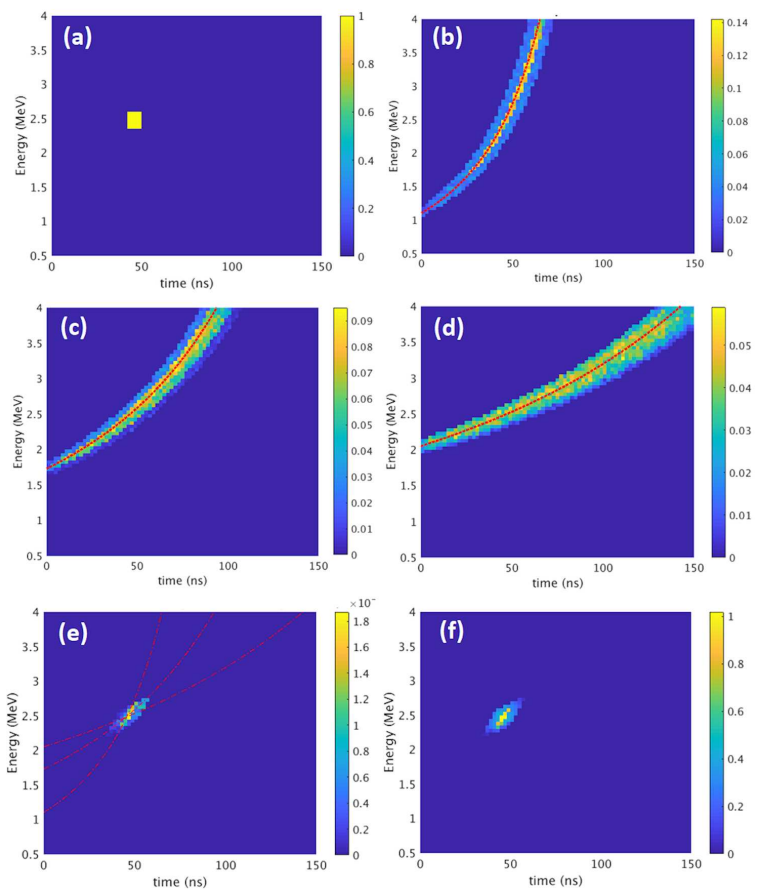

Fig. 8. (a) Original Dirac neutron spectrum and Monte Carlo reconstruction using one detector only placed at (b) $2 \mathrm{~m}$, (c) $5 \mathrm{~m}$, (d) $10 \mathrm{~m}$ from the source. (e) Hadamard multiplication of matrices shown in (b)-(d). (f) Reconstruction using the three detectors at once. The red lines in (b)-(e) correspond to the solution of the Dirac limit given by (11).

detectors at different distances allows to combine the information collected by the detectors and to restrict the reconstruction region as shown in Fig. 8e and $f$, increasing greatly the quality of the deconvolution process.

\subsection{Synthetic neutron spectrum deconvolution including perturbative noise}

The neutron spectrum deconvolution capabilities were investigated for a few synthetic cases and one typical example is reported here. This attempt was performed for a neutron spectrum composed of two emissivity blocks as depicted in Fig. 9. A perturbative $5 \%$ Gaussian noise, assumed to be independent of the signal level, was added in the synthetic measurements to test the robustness of the deconvolution process.

As a result, the MC algorithm was able to recover the two emissivity blocks of the original spectrum, despite a loss of resolution due to sparse measurements and perturbative noise. The quality of the reconstruction was further increased by using the smoothed reconstructed spectrum as a prior distribution probability in the MC loop, as presented in Fig. 10. The estimation of the validity of the method for different signal-to-noise ratios is kept as a perspective for future work. 


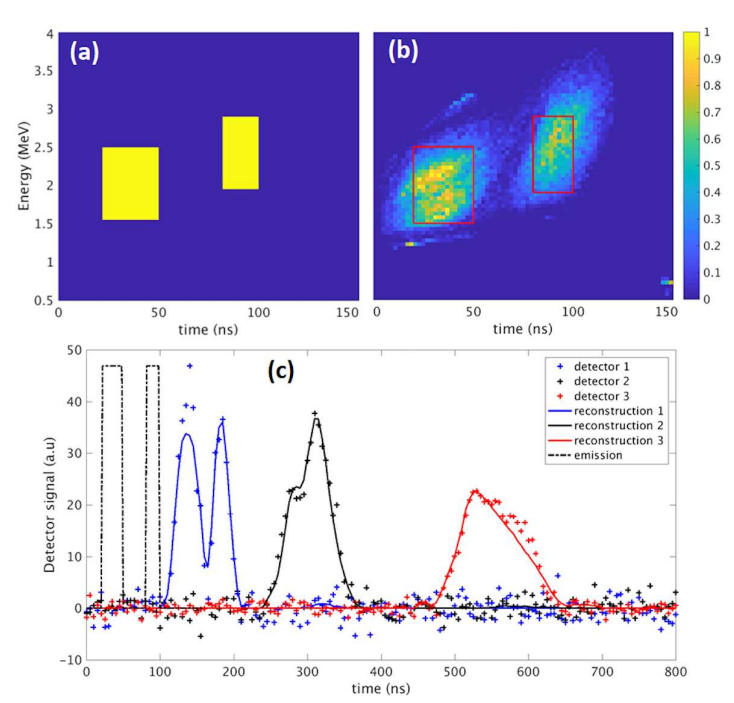

Fig. 9. (a) Original neutron spectrum. (b) Reconstructed spectrum after $20 \mathrm{MC}$ iterations and (c) associated ToF synthetic measurements of the detectors placed at $2 \mathrm{~m}, 5 \mathrm{~m}$, and $10 \mathrm{~m}$ from the PF-24.
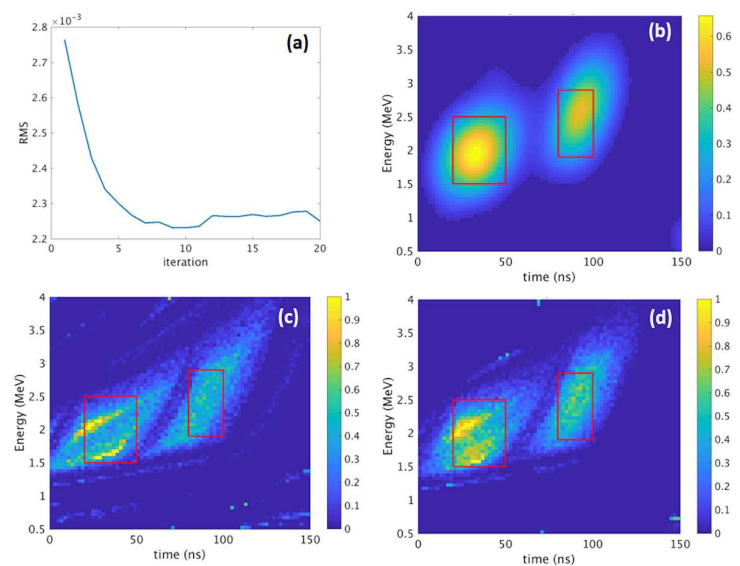

Fig. 10. (a) Global RMS error on the reconstructed spectrum after each MC iteration. (b) Gaussian-smoothed reconstructed spectrum used as prior probability distribution after a few MC iterations. (c) Reconstructed spectrum after $1 \mathrm{MC}$ iteration and $(\mathrm{d})$ after $3 \mathrm{MC}$ iterations.

\section{Conclusion}

The developed tomography method based on neural networks was tested against perturbative noise and with experimental SXR data from a Tore Supra discharge subject to tungsten impurity injection. The reconstructions are found to be consistent with the former work while speeding up the inversion process. The quality of the reconstruction could be further increased by using convolutional neuron layers [5], particularly adapted to image recognition problems, instead of fully-connected layers. In parallel, a Monte Carlo method for neutron spectrum deconvolution on the PF-24 from ToF measurements has been developed, in the prospect of experimental sessions foreseen on the PF-24 to assess the correlation between the pinch disruption, X-ray emission and emitted neutron spectrum. The method exhibits, despite a limited resolution due to the limited detector-source distance of $10 \mathrm{~m}$, robust reconstructions for the studied cases with synthetic data including perturbative noise in measurements. As a perspective for future work, the different inversion methods considered in this paper could be generalized to different fusion tomography diagnostics - like X-ray spatial tomography or neutron spectrum deconvolution - and benchmarked in order to associate an optimal method with each considered inversion problem.

\section{Acknowledgments}

This work has received financial support from the POLONIUM collaboration programme between the French ministries MEAE, MESRI and the Ministry of Science and Higher Education of Poland.

\section{References}

[1] M. Scholz, Plasma Focus and Controlled Nuclear Fusion, Institute of Nuclear Physics PAS, 2016.

[2] M. Anton, H. Weisen, M.J. Dutch, W. von der Linden, F. Buhlmann, R. Chavan, B. Marletaz, P. Marmillod, P. Paris, Plasma Phys. Control. Fusion 38, 1849 (1996).

[3] A. Jardin, D. Mazon, J. Bielecki, Phys. Scr. 91, 044007 (2016).

[4] J. Bielecki, J. Fusion Energy 37, 201 (2018).

[5] D.R. Ferreira, P.J. Carvalho, H. Fernandes, JET Contributors, Fusion Sci. Technol. 74, 47 (2018).

[6] A. Jardin, J. Bielecki, D. Mazon, J. Dankowski, K. Król, Y. Peysson, M. Scholz, Laser Part. Beams 37, 171 (2019).

[7] J. Mlynar, T. Craciunescu, D.R. Ferreira, P. Carvalho, O. Ficker, O. Grover, M. Imrisek, J. Svoboda, JET contributors, J. Fusion Energy 38, 458 (2019).

[8] J. Bielecki, A. Wojcik-Gargula, U. Wiacek, M. Scholz, A. Igielski, K. Drozdowicz, U. Woznicka, Eur. Phys. J. Plus 130, 145 (2015).

[9] J. Bielecki, A. Kurowski, J. Fusion Energy 38, 386 (2019).

[10] K. Rezac, D. Klir, P. Kubes, J. Kravarik, Plasma Phys. Control. Fusion 54, 105011 (2012).

[11] I.M. Ivanova-Stanik, R. Miklaszewski, Eur. Phys. J. D 54, 293 (2009). 